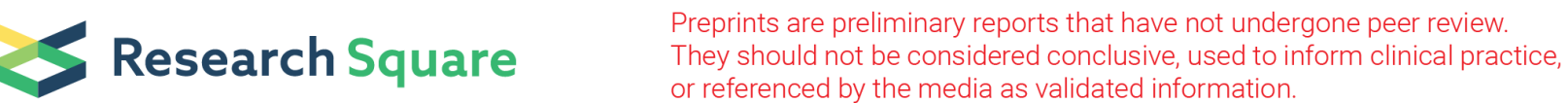

\section{F MRI-guided Flexible Low-intensity Focused Ultrasound for Drug Delivery and Molecular Targeted Therapy}

Jie Yang

the Fourth Hospital of Harbin Medical University

\section{Yingbo Li}

Fourth Affiliated Hospital of Harbin Medical University

Jiemei Sun

Fourth Affiliated Hospital of Harbin Medical University

Hongyan Zou

Fourth Affiliated Hospital of Harbin Medical University

\section{Qian Xie}

Fourth Affiliated Hospital of Harbin Medical University

\section{Rong A}

Fourth Affiliated Hospital of Harbin Medical University

Hongbin Wang

Fourth Affiliated Hospital of Harbin Medical University

\section{Xiaona Li}

Fourth Affiliated Hospital of Harbin Medical University

\section{Kai Wang}

Harbin Medical University

\section{Lili Yang}

Fourth Affiliated Hospital of Harbin Medical University

\section{Lina Wu}

Fourth Affiliated Hospital of Harbin Medical University

Xilin Sun ( $\square$ sunxl@ems.hrbmu.edu.cn)

Fourth Affiliated Hospital of Harbin Medical University https://orcid.org/0000-0001-7464-5117

\section{Article}

Keywords: perfluorocarbon, the third-generation EGFR-TKI, low-intensity focused ultrasound, on-demand drug release, tumor penetration

Posted Date: June 4th, 2021 
DOl: https://doi.org/10.21203/rs.3.rs-538349/v1

License: (c) (1) This work is licensed under a Creative Commons Attribution 4.0 International License. Read Full License 


\section{Abstract}

Non-site-specific, time-release and inefficient tumor penetration remain the major obstacles to the clinical efficacy of anticancer drugs. Novel strategies are therefore urgently needed for developing stimuliresponsive, local drug delivery systems that can increase drug penetration and accumulation at tumor sites. Here, we firstly describe an on-demand drug delivery system, AZD9291-PFCE nanoparticles (NPs) with ${ }^{19} \mathrm{~F}$ magnetic resonance imaging $\left({ }^{19} \mathrm{~F} \mathrm{MRI}\right)$-guided flexible low-intensity focused ultrasound (LIFU). Optimized AZD9291-PFCE NPs (size 73.9 $\pm 1.9 \mathrm{~nm}$ ) reach high accumulation in non-small cell lung cancer (NSCLC) tumor tissue. Further, LIFU triggered drug release from AZD9291-PFCE NPs and specifically increased tumor vascular and tumor tissue permeability. Quantitative ${ }^{19} \mathrm{~F}$ MRI was used to measure NPs accumulation in tumors in real-time after LIFU irradiation and to monitor therapeutic efficacy. Thus, we present an innovative strategy to achieve on-demand release of AZD9291 and improve NSCLC EGFR-targeted therapy efficacy by integrating theranostic NPs and ${ }^{19} \mathrm{~F}$ MRI-guided LIFU.

\section{Introduction}

Increasing drug delivery to tumors while reducing adverse side-effects is a key challenge in medical oncology, particularly in non-small cell lung cancer (NSCLC) ${ }^{1-3}$. Perfluorocarbon nanoparticle (PFC NP) delivery systems are a promising alternative to conventional chemotherapy drug administration. ${ }^{4-6}$ Drug molecules can be loaded onto the surface of PFC NPs to improve solubility and pharmacokinetics and ultimately be released after active or passive targeting to the tumor site, thereby considerably enhancing drug accumulation within tumor cells. ${ }^{7-9}$ Moreover, the core of PFCs can be detected by ${ }^{19} \mathrm{~F}$ magnetic resonance imaging $\left({ }^{19} \mathrm{~F} \mathrm{MRI}\right)$, a non-invasive molecular imaging modality in precision medicine, allowing the tracking of drug biodistribution and monitoring of treatment efficacy ${ }^{10-15}$. Although these PFC-based drug delivery systems (DDS) have a number of advantages, problems such as limited delivery efficiency and poor penetration are still main obstacles for preclinical and clinical cancer treatment ${ }^{16,17}$. Thus, the development of site-specific and time-release controlled PFC-based DDS is necessary to ensure efficient penetration of nanocarriers into deep tumor tissue ${ }^{18}$.

Low-intensity focused ultrasound (LIFU) is an effective approach for achieving spatiotemporal control of drug release at the desired site, leading to improved chemotherapy efficacy and reduced adverse sideeffects to healthy tissues. ${ }^{19-22}$ Other advantages of LIFU include its non-invasiveness, the absence of ionizing radiations, and the possibility to easily regulate tissue penetration depth by tuning frequency, duty cycles and time exposure of ultrasounds. ${ }^{23-25}$ Additionally, it has been shown that the mechanical effects derived from ultrasound waves can induce carrier destruction and drug release, as well as significantly increase cell membrane permeability, thereby facilitating cellular uptake of therapeutic agents throughout the entire tumor ${ }^{26,27}$. Thus, in recent years there has been growing interest in developing LIFU-triggered DDS for precise tumor targeting, increased tumor penetration, and more effective cancer therapy. ${ }^{28}$ 
Herein, we describe a novel ${ }^{19} \mathrm{~F}$ MRI-guided and LIFU-triggered DDS that achieves efficient delivery and deep penetration of nanocarriers for EGFR-targeted therapy in NSCLC (Fig. 1). Multimodal theranostic nanoplatforms were fabricated for the NP carrier by coating a perfluoro-15-crown-5-ether (PFCE) core with a lipid layer containing the new epidermal growth factor receptor tyrosine kinase inhibitor (EGFR-TKI) AZD9291. AZD9291 is a third-generation, irreversible, oral EGFR-TKI that selectively inhibits both EGFRTKI-sensitizing and EGFR-T790M resistance mutations ${ }^{29}$. We show that AZD9291-PFCE NPs retain the encapsulated drug under physiological conditions and release the payload quickly when triggered by LIFU, leading to increased accumulation and deeper penetration of AZD9291 into the tumor tissue. The biodistribution of NPs could be tracked with ${ }^{19} \mathrm{~F} \mathrm{MRI}$ in a quantitative manner. Furthermore, dynamic changes in cellular internalization, targeted real-time imaging, and AZD9291 concentration in tumors under LIFU sonication were all closely associated with treatment efficacy in vitro and in vivo. To summarize, in this study we achieved (i) the development of an intelligent DDS integrating ${ }^{19} \mathrm{~F} \mathrm{MRI}$ and EGFR-targeted therapy; (ii) monitoring of AZD9291 accumulation in tumors after LIFU irradiation with ${ }^{19} \mathrm{~F}$ MRI for precise guided therapy; and (iii) on-demand drug release triggered by LIFU with minimal systemic toxicity. Together these results strongly suggest that this novel approach is a promising treatment strategy with synergistic tumor-targeted theranostics capability.

\section{Results}

Synthesis and characterization of AZD9291-PFCE NPs. AZD9291-PFCE NPs were synthesized using microfluidization, similar to previous studies ${ }^{30-32}$. PFCE and AZD9291 were encapsulated simultaneously in the core and surface, respectively. AZD9291-PFCE NPs with nanoscale size could pass through the tumor vascular endothelial gap to the extravascular tumor tissue via active folic acid targeting, thus overcoming biological barrier for effective tumor treatment, which had been proved by our previous study ${ }^{33}$. Transmission electron microscopy (TEM) images revealed that AZD9291-PFCE NPs have spherical morphology with uniform particle size and distribution (Fig. 2a). To reveal the composition of AZD9291-PFCE NPs, the corresponding element mappings of carbon (C), nitrogen (N), oxygen (O), and fluorine (F) exhibit the homogeneous distribution of each element in the framework of NPs, further confirming that AZD9291 and PFCE were loaded in the NPs (Fig. 2b). The C, 0 and F intensities of PFCE NPs were shown in Supplementary Fig. 1. The average hydrodynamic size of AZD9291-PFCE NPs was $73.9 \pm 1.9 \mathrm{~nm}$ (Fig. 2C). As shown in the photograph inset in Fig. 2c, AZD9291-PFCE NPs produced an emulsion with a light purple appearance after dissolving in deionized water. The average hydrodynamic size of PFCE NPs was $100.9 \pm 8.2 \mathrm{~nm}$ (Supplementary Fig. 2). Compared with the surface charge of PFCE NPs $(-18.4 \pm 0.5 \mathrm{mV})$, the zeta potential of AZD9291-PFCE NPs was $46.7 \pm 1.3 \mathrm{mV}$ (Supplementary Fig. 3). Importantly, no significant size changes were observed for 8 weeks at $4{ }^{\circ} \mathrm{C}, 25^{\circ} \mathrm{C}$ or $37^{\circ} \mathrm{C}$ storage, demonstrating the excellent stability of AZD9291-PFCE NPs (Fig. 2d). To assess encapsulation efficiency of AZD9291-PFCE NPs, AZD9291 was extracted and measured using HPLC. Encapsulation efficiency was 87.38\%, showing that PFCE NPs are an appropriate vehicle to efficiently encapsulate AZD9291. 
Given their nontoxicity and ability to modify reactive surfaces ${ }^{34}$, rhodamine-phospholipids were embedded in PFCE NPs to improve imaging efficacy and early diagnosis with ${ }^{19} \mathrm{~F}-\mathrm{MR}$ and NIRF imaging ${ }^{35} .{ }^{19} \mathrm{~F}$ MR images and quantitative analyses showed that significantly higher signal was consistently obtained with increasing concentrations of AZD9291-PFCE NPs (Fig. 2e, f). Compared with PFCE NPs, AZD 9291-PFCE NPs exhibited the characteristic absorption peak of AZD9291 at $335 \mathrm{~nm}$, indicating successful loading of the drug (Fig. 2g). Furthermore, the UV-Vis absorption spectra of AZD9291-PFCE NPs embedded with rhodamine-PE displayed a small absorption peak at $570 \mathrm{~nm}$. Finally, fluorescence images of AZD9291-PFCE NPs showed a clear correlation with ${ }^{19} \mathrm{~F}$ MRI results (Supplementary Fig. 4).

LIFU-triggered on-demand drug release. Controlled release systems are commonly used to regulate drug release and transport drugs across physiological barriers to designated areas while reducing drug exposure in healthy tissues ${ }^{36-38}$. We found that drug release from NPs occurs relatively faster at $\mathrm{pH}$ 5.0, when compared to $\mathrm{pH} 6.5$ and $\mathrm{pH} 7.4$ (Fig. 2h). Furthermore, drug-release behavior under LIFU irradiation was assessed at $\mathrm{pH}$ 7.4. While drug release from AZD9291-PFCE NPs occurred slowly in the absence of LIFU irradiation, the cumulative release rate increased rapidly after LIFU irradiation of the nanoemulsion (Fig. 2i). LIFU-responsive drug release can be attributed to the mechanical effect of ultrasounds, which activate water and drug molecules diffusion. Furthermore, ultrasounds may also disrupt the structure of phospholipid surfactants, resulting in the release of the entrapped drugs. These results demonstrate that LIFU is a valuable method to enhance drug release from AZD9291-PFCE NPs and improve therapy efficacy.

LIFU enhanced cellular internalization and penetration capabilities of AZD9291-PFCE NPs. To determine whether LIFU improves cellular uptake of NPs, H1975 cells were incubated with rhodamine-labeled PFCE NPs and AZD9291-PFCE NPs and then exposed to LIFU irradiation. Confocal laser scanning microscopy (CLSM) images showed significantly higher cellular uptake of NPs after $4 \mathrm{~h}$ of incubation under LIFU irradiation than in the absence of LIFU irradiation (Fig. 3a). Measurements of mean fluorescence intensity confirmed this result (Fig. 3b, c). To further investigate whether LIFU irradiation can effectively promote the penetration of NPs in tumors, three-dimensional (3D) tumor spheroid models were used for evaluating in vitro penetration. ${ }^{39}$ Compared to $2 \mathrm{D}$ monolayer cell culture systems, 3D tumor spheroids are closer to real tumor environment which can simulate tissue for cell-cell connections. ${ }^{40}$ Thus, 3D tumor spheroids are an ideal model for assessing the permeability of NPs in vitro and can offer valuable reference for in vivo evaluation. ${ }^{41} \mathrm{H} 1975$ 3D tumor spheroids were established and observed by CLSM when the diameter reached to about $400 \mu \mathrm{m}$. As shown in Fig. 3d, the penetration of PFCE NPs and AZD9291-PFCE NPs without LIFU irradiation was distributed only in the surface layer of the tumor spheroids. On the contrary, PFCE NPs and AZD9291-PFCE NPs penetrated throughout the entire spheroid under LIFU irradiation. The intensity and distribution of fluorescence could be clearly observed via 3D diagram of $80 \mu \mathrm{m}$ depth sections reconstructed by Image $J$ (Fig. 3e). These findings suggest that LIFU enhances cellular internalization and tumor penetration capabilities thus allowing entry of the NPs into H1975 cells. 
Synergistic antitumor effects in vitro. To assess the biocompatibility of PFCE NPs, MTT assays were conducted in $\mathrm{H} 1975$ and $\mathrm{H} 520$ cells incubated with various concentrations of the formulations. Cell viability was not affected by PFCE NPs at any concentration up to $10 \mathrm{mg} / \mathrm{mL}$ (Supplementary Fig. 5a, b), suggesting that perfluorocarbon NPs are suitable for in vivo applications. However, cell viability of H1975 and H520 cells declined significantly with increasing concentrations of AZD9291-PFCE NPs and free AZD9291, as well as with treatment time (Fig. 4a, b and Supplementary Fig. 6a, b). Furthermore, AZD 9291-PFCE NPs and free AZD 9291 exhibited greater potency in H1975 cells (L858R/T790M) than in H520 cells (wild-type EGFR) (Supplementary Table 1), consistent with previous studies ${ }^{42}$. Interestingly, at the same AZD9291 concentration, the cytotoxicity of cells treated with free AZD9291 was higher than that of cells treated with AZD9291-PFCE NPs. While free AZD9291 is highly susceptible to entry into cells by passive diffusion and internalization, AZD9291 in NPs is released gradually into the surrounding media, explaining the relative increase in cytotoxicity over time in vitro ${ }^{43}, 44$. Notably, the cell viability of cells treated with AZD9291-PFCE NPs or free AZD9291 decreased significantly after LIFU irradiation (Fig. 4c). Importantly, no noteworthy cytotoxicity was detected in $\mathrm{H} 1975$ cells in either the LIFU irradiationonly or PFCE NPs with LIFU group, showing that the LIFU irradiation dose used presents no obvious toxicity and is safe for cells. The remarkable cytotoxicity of AZD9291-PFCE NPs when combined with LIFU likely results from a combination of increased cell membrane permeability and LIFU-responsive enhanced release of AZD9291 at the target site, which leads to a considerably improvement of the antitumor efficacy of the NPs. To further explore the synergistic efficacy of AZD9291-PFCE NPs and LIFU, flow cytometry and TUNEL assays were conducted to evaluate cell apoptosis. Consistent with the MTT results, LIFU combined with AZD9291-PFCE NPs or free AZD9291 increased apoptosis in H1975 cells, particularly AZD9291-PFCE NPs with LIFU, further suggesting that LIFU enhances apoptotic effect (Fig. 4d-f). These results show that AZD9291-PFCE NPs combined with LIFU irradiation have superior therapeutic efficacy in H1975 cells. And PFCE NPs have high biocompatibility and LIFU irradiation dose is safe for cells without obvious toxicity.

${ }^{19} \mathrm{~F}$ MRI monitored penetration and accumulation of NPs in tumor. To assess the feasibility of using AZD 9291-PFCE NPs to monitor therapeutic efficacy in vivo, NP penetration and accumulation in tumors were visualized on a MRI-compatible LIFU system using a single-focus transducer that achieves tumor coverage with high flexibility (Fig. 5a). After injection of $300 \mu \mathrm{L}$ of PFCE NPs or AZD9291-PFCE NPs into $\mathrm{H} 1975$ xenograft-bearing nude mice through the tail vein, the NPs in tumors were identified and quantified with ${ }^{19} \mathrm{~F}$ MRI. $4 \mathrm{~h}$ after injection, the mice were subjected to LIFU irradiation $\left(1.1 \mathrm{MHz}, 55.8 \mathrm{~mW} / \mathrm{cm}^{2}, 50 \%\right.$ duty cycle, pulse wave mode, $3 \mathrm{~W}, 20 \mathrm{~min}$ ) on an area covering the tumor. MRI was conducted during irradiation to provide real-time feedback. The anatomical images were observed by ${ }^{1} \mathrm{H}$ MRI (Supplementary Fig. 7). As shown in Fig. 5b, ${ }^{19} \mathrm{~F}-\mathrm{MR}$ signal intensity was detected in the tumor area 1 48 $\mathrm{h}$ after injection of PFCE NPs or AZD9291-PFCE NPs with LIFU assistance, peaking at $48 \mathrm{~h}$. Quantitative analyses were consistent with the ${ }^{19} \mathrm{~F}-\mathrm{MRI}$ results (Fig. $5 \mathrm{c}$ ). $48 \mathrm{~h}$ after injection, intra-tumoral concentrations of AZD9291-PFCE NPs was 2.6-fold $(P<0.001)$ higher with LIFU irradiation than without. These data demonstrate that irradiation with LIFU improved the tumor-penetrating efficiency of NPs in 
vivo, thereby enhancing their antitumor activity. Thus, ${ }^{19} \mathrm{~F}$ MRI is a valuable technique to support real-time monitor after local LIFU application.

${ }^{19} \mathrm{~F}$ MRI-guided flexible LIFU effectively delivered NPs to tumor. AZD9291 content in tumor tissues and normal organs was measured by HPLC at $48 \mathrm{~h}$ after treatment with or without LIFU (Fig. 5d). AZD9291PFCE NP accumulation in tumor was 1.8 times higher $(P<0.01)$ than that of free AZD9291. Furthermore, with LIFU irradiation AZD9291-PFCE NP accumulation in tumor was 1.3-fold $(P<0.05)$ higher than without LIFU. These results show that AZD9291-PFCE NPs with LIFU irradiation significantly increase the drug accumulation in tumors. To confirm the above ${ }^{19} \mathrm{~F}$ MRI quantitative analysis, tissue biodistribution of fluorescent PFCE NPs and AZD9291-PFCE NPs was assessed in mice using a Xenogen IVIS imaging system at $48 \mathrm{~h}$ after administration (Supplementary Fig. 8). Notably, the rodents eliminated the NPs rapidly into the small intestine through the biliary system ${ }^{45}$. Furthermore, CLSM analysis of tissue sections made $48 \mathrm{~h}$ after the injection revealed that the fluorescence intensity of PFCE NPs and AZD 9291-PFCE NPs in tumor was significantly stronger with LIFU than in the absence of LIFU irradiation (Fig. 5e, f). In the majority of normal tissues, no obvious difference in fluorescence intensity was detected between NPs with or without LIFU (Supplementary Fig. 9), indicating that LIFU enhances the penetrability of NPs into tumors but not normal tissues. Finally, a positive correlation was found between AZD9291 content measured by HPLC, the fluorescence intensity of NPs and ${ }^{19} \mathrm{~F}$ concentration in tumor tissues (Fig. 5g). Overall, these results strongly support the potential applicability of ${ }^{19} \mathrm{~F}$ MRI-guided LIFU for delivering AZD9291-PFCE NPs locally to tumor sites in a noninvasive manner.

AZD9291-PFCE NPs combined with LIFU significantly suppressed tumor growth. Given the remarkable therapeutic efficacy of AZD9291-PFCE NPs and LIFU in vitro, the in vivo antitumor efficacy of this combination treatment was investigated in $\mathrm{H} 1975$ subcutaneous tumor models. Antitumor effect was evaluated following the treatment schedule indicated in Fig. 6a. After treatment, changes in tumors with an initial volume of $100 \sim 200 \mathrm{~mm}^{3}$ were tracked by ${ }^{1} \mathrm{H}$ MRI (Fig. 6b). Digital photographs of different groups of mice were taken to show the treatment effect (Supplementary Fig. 10). Therapeutic efficacy was assessed by monitoring changes in tumor volume and weight, and tumor growth inhibition (TGI). During the treatment, the tumor volume of control and PFCE NPs groups increased rapidly. However, treatment with free AZD9291 or AZD9291-PFCE NPs suppressed the growth of xenografts (Fig. 6c), and this effect was stronger after LIFU irradiation, particularly in the AZD9291-PFCE NPs group. No significant differences were found between the control groups with or without LIFU $(P>0.05)$, suggesting that the LIFU irradiation dose was safe. On day 14, the tumor weights in free AZD9291 or AZD9291-PFCE NPs groups were approximately $39.24 \%$ and $16.71 \%$ of the control tumor weight, respectively (Fig. $6 \mathrm{~d}$ ), and LIFU further improved the antitumor effect. Indeed, tumor weight in the AZD9291-PFCE NPs with LIFU group was only $2.04 \%$ of the control tumor weight, which is remarkably low even when compared to the AZD 9291 with LIFU group (27.63\%). The isolated tumors were shown in Supplementary Fig. 11a. TGI measurements were consistent with these results (Supplementary Fig. 11b). The implementation of LIFU enhanced the intratumoral accumulation and distribution of the drug inhibiting tumor proliferation. Interestingly, these in vivo results show that free AZD9291 exhibits lower therapeutic efficacy than 
AZD9291-PFCE NPs, which is inconsistent with the proliferation results in vitro. As the drugs were administered systemically, there is lower accumulation of free AZD9291 in the tumor region likely due to the poor solubility and rapid hydrolysis of free AZD9291 in vivo. Thus, the therapeutic effect of free AZD9291 was not satisfactory in vivo. Furthermore, encapsulating AZD9291 in the NPs enhances stability, decreases toxicity, increases AZD9291 concentration at the tumor site, and prolongs the action of AZD9291 in vivo by controlled release.

The survival rate of mice administrated with different treatment groups is shown in Fig. 6e. AZD9291PFCE NPs with LIFU treatment significantly extended survival to 60 days. As shown in Fig. $6 f$, there were also no obvious differences in body weight during the treatment course in all groups, indicating that neither the NPs or LIFU irradiation cause toxicity. To determine the histological characteristics of lung tumor tissues after treatment, H\&E, Ki67, CD31 and TUNEL staining were carried out (Fig. 6g). H\&E staining revealed that large amounts of tumor cells were destroyed, demonstrating the remarkable therapeutic efficacy of AZD9291-PFCE NPs with LIFU assistance. Ki67 expression was absent or reduced in tumor tissues of the AZD9291-PFCE NPs with LIFU group, contrary to tumor tissues in other groups (Supplementary Fig. 12a). Low CD31 expression in mice treated with AZD9291-PFCE NPs and LIFU revealed that the treatment causes reduced tumor microvascular density (Supplementary Fig. 12b). TUNEL staining showed that apoptosis was increased in tumors of mice treated with AZD9291-PFCE NPs and LIFU. To investigate the molecular mechanism of anticancer activity, we performed phosphorylatedEGFR (pEGFR) immunohistochemistry to assess the molecular changes after treatment. pEGFR expression was significantly declined in tumor tissues of the AZD9291-PFCE NPs with LIFU group. These results indicate that AZD9291-PFCE NPs can efficiently penetrate the cell membrane with LIFU assistance, leading to improved antitumor therapeutic efficacy in H1975 tumor-bearing mice.

Safety evaluation. To assess the biological safety of LIFU combined with AZD9291-PFCE NPs in tumor therapy in vivo, H\&E staining was conducted on the major organs (heart, liver, spleen, lung, kidney, muscle and intestine), and electrolytes and blood biochemical/hematology indexes were determined. As expected, no cell-integrity damage, necrosis or inflammatory reactions were observed on H\&E-stained sections of normal organs in all the treatment groups, demonstrating the excellent biocompatibility of the NPs (Fig. 7a). No significant differences in the hematology parameters were detected on days 1 and 7 after intravenous injection with PFCE NPs or AZD9291-PFCE NPs, when compared to the control group (Fig. 7b). These results indicate that intravenous injection of PFCE NPs and AZD9291-PFCE NPs at a dose of $300 \mu \mathrm{L}$ per mouse is feasible and safe. Furthermore, no significant differences in systemic toxicity parameters (RBC, PLT, WBC, ALT, AST, BUN and Crea) were found between the AZD9291-PFCE NPs with LIFU and control groups (Fig. 7c-e). However, AST and BUN were increased after treatment with free AZD9291 or free AZD9291 with LIFU, suggesting that free AZD9291 causes side effects in the liver and kidney. These results indicate that AZD9291 loaded on NPs is less toxic than free AZD9291. Indeed, LIFU-irradiated AZD9291-PFCE NPs release AZD9291 at the tumor site, which likely reduces systemic toxicity. These findings suggest that AZD9291-PFCE NPs combined with LIFU is a promising strategy for targeted delivery of AZD9291 to lung cancer, significantly improving treatment efficiency with no severe side effects. 


\section{Discussion}

There is an urgent need for therapeutics and delivery methods that increase drug penetration and accumulation at tumor sites. In this study, we developed a synergistic strategy combining AZD9291-PFCE NPs with LIFU assistance to enhance the delivery of AZD9291 into tumors. AZD9291-PFCE are perfluoro15-crown-5-ether nanoparticles (NPs) loaded with the third-generation epidermal growth factor receptor (EGFR) tyrosine kinase inhibitor AZD9291. The water-solubility of AZD9291 with PFC NPs as the carrier was greatly improved when compared to free AZD9291. Owing to their nanoscale properties and LIFUtriggered controlled release activity, this platform provides advantages over existing delivery technologies in terms of 1) sustained release of the therapeutic NPs; 2) on-demand drug release and increased tumorpenetrating efficiency through mechanical effect induced by LIFU irradiation; 3) therapeutic efficacy achieved through multistage drug delivery; and 4) visualization of NPs quantities in tumor based on ${ }^{19} \mathrm{~F}$ MRI technology. This work also elucidated important relationships between targeted real-time imaging, the concentration of AZD9291 in tumor and treatment efficacy, highlighting that ${ }^{19} \mathrm{~F}$ MRI is a valuable tool for accurately assessing targeted therapeutic effects with LIFU assistance, ultimately contributing to the optimization of treatment procedures. Importantly, AZD9291-PFCE NPs can be administered through intravenous routes and be locally triggered by LIFU at the tumor site to achieve multistage drug delivery for therapeutic benefit, potentially opening new clinical opportunities for leveraging this system. Currently, high systemic toxicity is a key challenge in using AZD9291 for cancer patient care. Here, biosafety analyses of AZD9291-PFCE NPs revealed excellent clinical translational potential. Thus, we have shown that AZD9291-PFCE NPs with ${ }^{19} \mathrm{~F}$ MRI-guided flexible LIFU assistance may represent a significant technological advancement to augment delivery efficiency of AZD9291 and improve the therapeutic effect of NSCLC.

\section{Methods}

Materials. Perfluoro-15-crown-5 ether (PFCE, $\mathrm{C}_{10} \mathrm{~F}_{20} \mathrm{O}_{5}$ ) was purchased from Exfluor Research Corporation (TX, USA). High-purity lecithin, cholesterol and 2-dipalmitoyl-snglycero-3-phosphoethanolamine-N(lissaminerhodamine B sulfonyl) (16:0 LissRhod PE) were purchased from Avanti Polar Lipids, Inc. (AL, USA). AZD9291 was purchased from Medchem Express (NJ, USA). Glycerin was purchased from Aldrich Chemical Co. (WI, USA). Argon was used for the storage of materials. The Spectra/Por membrane (Cellulose MWCO: $20 \mathrm{kDa}$ ) used for dialysis was purchased from Spectrum Medical Industries, Inc. (CA, USA). Zoletil ${ }^{\mathrm{TM}} 50$ was obtained from Virbac (Nice, France). All chemicals used in this work were of analytical grade and used as received.

Preparation of AZD9291-PFCE nanoparticles. AZD9291-PFCE NPs were synthesized by microfluidization method according to the procedure reported previously. ${ }^{30-32}$ Briefly, the nanoparticulate emulsions were comprised of $20 \%(\mathrm{v} / \mathrm{v})$ PFCE, $3 \%(\mathrm{w} / \mathrm{v})$ of a surfactant commixture, $2.5 \%(\mathrm{w} / \mathrm{v})$ glycerin and water for the balance. The surfactant co-mixture of NPs included $73 \mathrm{~mol} \%$ lecithin, $14.9 \mathrm{~mol} \%$ cholesterol, $12 \mathrm{~mol} \%$ AZD9291, 0.1 mol\% 1,2-dipalmitoyl-sn-glycero-3-phosphoethanolamine-N-(lissaminerhodamine B 
sulfonyl) (ammonium salt) (16:0 LissRhod PE), and 0.05 mol\% 1,2-dipalmitoyl-sn-glycero-3phosphoethanolamine-N-(6-((folate)amino)hexanoyl) (sodium salt) (16:0 Folate Cap PE) on the outer shell to endow an FR targeting. The surfactant components were combined with PFCE, water and glycerin, then homogenized at 20,000 psi for 4 min with a microfluidizer M110P (Microfluidics, Newton, $\mathrm{MA}$ ). Procedures were made following dialysis (20 kDa MWCO cellulosic membrane) of nanoparticle suspensions into deionized water. The resulting emulsions were conserved under argon in aseptic sealed vials until use.

Characterization of nanoparticles. Transmission electron microscopy (TEM) images and elemental mapping analysis were characterized using a FEI Talos F200X instrument equipped with 4 Super-X SDDs. The size distribution and zeta potential of NPs were measured using a Zetasizer Nano ZS90 (Malvern Instruments). The absorption spectra were determined by Perkin-Elmer Lambda 35 UV/Vis spectrophotometer (Perkin-Elmer, USA). To evaluate the imaging properties of AZD9291-PFCE NPs, the phantoms with various ${ }^{19} \mathrm{~F}$ concentrations were investigated by ${ }^{19} \mathrm{~F}$ MRI and fluorescence imaging. The AZD9291 concentration in AZD9291-PFCE NPs was examined by high performance liquid chromatography (HPLC). The encapsulation efficiency (EE) of AZD9291-PFCE NPs was calculated as follows:

$\mathrm{EE} \%=\frac{\text { weight of drug encapsulated in nanoparticles }}{\text { initial weight of drug }} \times 100 \%$

In vitro drug release. The release property of AZD9291-PFCE NPs was determined using a dialysis method at different $\mathrm{pH}$ values. Briefly, $1 \mathrm{~mL}$ of AZD9291-PFCE NPs was sealed in a dialysis bag (MWCO $3000 \mathrm{Da}$ ) and immersed into $400 \mathrm{~mL}$ of phosphate buffered saline (PBS) solution (pH 5.0, 6.5 and 7.4 ) at $37^{\circ} \mathrm{C}$ with continuous shaking. At the time points of $1 \mathrm{~h}, 2 \mathrm{~h}, 4 \mathrm{~h}, 8 \mathrm{~h}, 12 \mathrm{~h}, 24 \mathrm{~h}, 48 \mathrm{~h}$ and $72 \mathrm{~h}, 1 \mathrm{~mL}$ of release medium was collected and replaced by $1 \mathrm{~mL}$ of fresh buffer to maintain the sink condition. To investigate the LIFU-triggered drug release profile of AZD9291-PFCE NPs, $1 \mathrm{~mL}$ of AZD9291-PFCE NPs were injected into dialysis bag with LIFU irradiation and dialyzed against $400 \mathrm{~mL}$ of PBS (pH 7.4) at $37^{\circ} \mathrm{C}$ in a shaking water bath. An acoustic frequency of $1.1 \mathrm{MHz}$, an acoustic intensity of $55.8 \mathrm{~mW} / \mathrm{cm}^{2}$, a $50 \%$ duty cycle, pulse wave mode, a power of $3 \mathrm{~W}$ and a duration of 10 min were set as LIFU parameters for sonication. The next steps were similar to without LIFU group. The AZD9291 concentration in the collected buffer was measured by HPLC.

Cell culture and animal model. Human non-small cell lung cancer (NSCLC) cell lines, H1975 (EGFR L858R/T790M positive) and H520 (EGFR negative) were purchased from the American Type Culture Collection (ATCC). All cell lines were cultured in RPMI-1640 medium containing 10\% fetal bovine serum (FBS), penicillin $(100 \mathrm{U} / \mathrm{mL})$ and streptomycin $(100 \mu \mathrm{g} / \mathrm{mL})$. Cells were maintained in a humidified incubator with $5 \% \mathrm{CO}_{2}$ at $37^{\circ} \mathrm{C}$. Female BALB/c nude mice (5-6 weeks) were purchased from the Beijing Vital River Laboratory Animal Technology Co., Ltd. All animal studies were approved by the Institutional 
Animal Care and Use Committee of the Harbin Medical University and in accordance with all related ethical guidelines. The female BALB/c nude mice were inoculated subcutaneously into the right thigh with $200 \mu \mathrm{L}$ of cell suspension containing $1 \times 10^{7} \mathrm{H} 1975$ cells. The tumors were allowed to reach $100 \sim$ $200 \mathrm{~mm}^{3}$ before experimentation. The tumor volumes were calculated by the following equation: volume $=\left(\right.$ length $\times$ width $\left.^{2}\right) / 2$. Relative tumor volumes were calculated as V/VO (V0 was the tumor volume when the treatment was initiated).

Cellular uptake analysis. $\mathrm{H} 1975$ cells $\left(10^{5}\right.$ cells $\left./ \mathrm{mL}\right)$ were seeded in 6-well plates and allowed to grow until $80 \%$ confluence. Then PFCE NPs or AZD9291-PFCE NPs labeled with rhodamine B were added at a final concentration of $3 \mathrm{mg} / \mathrm{mL}$ of PFCE. LIFU $\left(55.8 \mathrm{~mW} / \mathrm{cm}^{2}, 50 \%\right.$ duty cycle, pulse wave mode, $3 \mathrm{~W}, 10$ min) was performed $1 \mathrm{~h}$ after incubation, respectively. After incubated for $4 \mathrm{~h}$ at $37^{\circ} \mathrm{C}$, the cells were washed with phosphate-buffered saline (PBS) twice and fixed with $4 \%$ paraformaldehyde for $10 \mathrm{~min}$. The cell nuclei were stained with 4', 6-diamidino-2-phenylindole (DAPI). Cell images were observed using a confocal laser scanning microscope (CLSM, Nikon, Japan).

Penetration in solid tumor spheroid. The three-dimensional (3D) tumor spheroids were established according to the reported protocol. ${ }^{39}$ Briefly, $\mathrm{H} 1975$ cells $\left(5 \times 10^{4}\right.$ cells $\left./ \mathrm{mL}\right)$ were seeded into 96 -well plates pre-coated with $1.7 \%$ low melting point agarose and incubated for a few days. When the diameter of tumor spheroids reached to about $400 \mu \mathrm{m}$, rhodamine-labeled PFCE NPs or AZD9291-PFCE NPs were added into the spheres with or without LIFU irradiation $\left(55.8 \mathrm{~mW} / \mathrm{cm}^{2}, 50 \%\right.$ duty cycle, pulse wave mode, $3 \mathrm{~W}, 10 \mathrm{~min}$ ). After incubation for $4 \mathrm{~h}$ at $37^{\circ} \mathrm{C}$, the tumor spheroids were washed by PBS and fixed with $4 \%$ paraformaldehyde for $15 \mathrm{~min}$, respectively. The Z-stack scanning for the spheroids was performed at $10 \mu \mathrm{m}$ intervals by CLSM.

Cell viability assay. The cell viabilities were evaluated by MTT assay. Briefly, $\mathrm{H} 1975$ and $\mathrm{H} 520$ cells were seeded in 96-well plates at the density of $1 \times 10^{4}$ cells/well and incubated for $24 \mathrm{~h}$. The experiment is divided into two parts. The first part was used to evaluate the cell viability of the $\mathrm{H} 1975$ and $\mathrm{H} 520$ cells treated with PFCE NPs, AZD9291 and AZD9291-PFCE NPs at different concentrations for $24 \mathrm{~h}$ and $48 \mathrm{~h}$. The second part was used to assess the cytotoxicity of the H1975 cells incubated with free AZD9291 solution and various formulations of NPs with or without LIFU irradiation for $24 \mathrm{~h}$. Part II included 8 groups: Control, PFCE NPs, AZD9291, AZD9291-PFCE NPs, Control + LIFU, PFCE NPs + LIFU, AZD9291 + LIFU and AZD9291-PFCE NPs + LIFU $(n=3)$. The groups of free AZD9291 and AZD9291-PFCE NPs had the same concentration of AZD9291 (0.1 MM). LIFU was carried out after incubation for $1 \mathrm{~h}$ with different treatments $\left(1.1 \mathrm{MHz}, 55.8 \mathrm{~mW} / \mathrm{cm}^{2}, 50 \%\right.$ duty cycle, pulse wave mode, $\left.3 \mathrm{~W}, 15 \mathrm{~min}\right)$. After incubation for $24 \mathrm{~h}$ and $48 \mathrm{~h}, 20 \mu \mathrm{L}$ MTT (3-(4,5-dimethylthiazol-2-yl)-2,5-diphenyltetrazolium bromide) solutions (5 $\mathrm{mg} / \mathrm{mL}$ ) were added to each well and incubated for $4 \mathrm{~h}$. After replacing the culture medium with $150 \mu \mathrm{L}$ dimethyl sulfoxide (DMSO), the optical density (OD) values were measured at $490 \mathrm{~nm}$ using a Synergy HTX microplate reader (Bio Tek, USA). All experiments were performed at least three times independently. The $\mathrm{IC}_{50}$ of $24 \mathrm{~h}$ and $48 \mathrm{~h}$ in different groups was calculated by GraphPad Prism 8.0 software. 
Cell apoptosis assay. $\mathrm{H} 1975$ cells were seeded in 6-well plates at a density of $5 \times 10^{5} \mathrm{cells} / \mathrm{mL}$ and cultivated in a humidified atmosphere at $37^{\circ} \mathrm{C}$ with $5 \% \mathrm{CO}_{2}$ for $24 \mathrm{~h}$. The grouping method of cellular apoptosis analysis was in accordance with the part II of cell viability assay. LIFU was applied at $1 \mathrm{~h}$ after different treatments with the same parameters described above. After $24 \mathrm{~h}$, the cells were harvested, centrifuged at $1000 \mathrm{~g}$ for $5 \mathrm{~min}$, washed with cold PBS twice and resuspended in binding buffer. Afterwards, $5 \mu \mathrm{L}$ of Annexin V-FITC and $5 \mu \mathrm{L}$ of PI were added and incubated with the cells for $15 \mathrm{~min}$ in the dark at room temperature. The stained cells were measured using MoFlo XDP flow cytometer (Beckman Coulter, Fullerton, CA, USA). The results were analyzed using Summit 5.2 analysis software (Beckman Coulter).

The apoptotic cells were also detected by TdT-mediated dUTP nick end labeling (TUNEL, Roche, Switzerland) staining. Briefly, cells were cultured in 6-well plates before various treatment, then fixed with $4 \%$ paraformaldehyde for $20 \mathrm{~min}$, and incubated with the TUNEL reaction mixture for $1 \mathrm{~h}$ at $37^{\circ} \mathrm{C}$. The number of TUNEL-positive cells was observed by CLSM.

In vivo MRI-guided LIFU. Mice were anesthetized with $2 \%$ isoflurane and injected with PFCE NPs and AZD 9291-PFCE NPs via the tail vein $(300 \mu \mathrm{L}, \mathrm{n}=3)$. The MRI-guided LIFU system is illustrated in Figure S8, Supporting Information. Mice were imaged with a 9.4 T MR scanner (BioSpec 94/20 USR; Bruker Biospin, Germany) using a ${ }^{1} \mathrm{H} /{ }^{19} \mathrm{~F}$ double tune volume coil and their respiration rate was monitored during MR scanning. Proton anatomical images were acquired with an axial T1-weighted rapid acquisition with relaxation enhancement (RARE) sequence and the following parameters: repetition time $(T R) /$ echo time $(T E)=820 / 12 \mathrm{msec}$, number of averages $(N A)=8$, RARE factor $=8$, matrix $=256 \times 256$, slice thickness $(\mathrm{ST})=1 \mathrm{~mm}$, and field of view $(\mathrm{FOV})=38.4 \times 38.4 \mathrm{~mm}^{2} .{ }^{19} \mathrm{~F}$ MRI was implemented with a RARE sequence $(T R / T E=2000 / 100 \mathrm{msec}, \mathrm{NA}=128$, RARE factor $=32$, matrix $=64 \times 64, \mathrm{ST}=3 \mathrm{~mm}, \mathrm{FOV}=$ $38.4 \times 38.4 \mathrm{~mm}^{2}$ ). A reference phantom with $44.38 \mathrm{mg} / \mathrm{mL}$ of PFC emulsion in agarose gel was put beside the tumor for signal quantification. The ${ }^{19} \mathrm{~F}$ image were displayed by pseudo-color processing and overlaid on corresponding ${ }^{1} \mathrm{H}$ anatomic images. ${ }^{19} \mathrm{~F}$ signal intensity was measured by the ParaVision 6.0.1 software and the fluorine concentration of tumors was calculated according to the determined concentration of the reference tube. Four hours after injection of NPs, LIFU $\left(1.1 \mathrm{MHz}, 55.8 \mathrm{~mW} / \mathrm{cm}^{2}, 50 \%\right.$ duty cycle, pulse wave mode, $3 \mathrm{~W}, 20 \mathrm{~min}$ ) was applied to trigger the location of the tumor and MRI was implemented synchronously. The permeability of PFCE NPs and AZD9291-PFCE NPs with LIFU irradiation was visualized and quantified by ${ }^{19} \mathrm{~F}$ MRI.

Biodistribution studies. 48 hours after administration of rhodamine-labeled PFCE NPs and AZD9291PFCE NPs with or without LIFU irradiation $\left(1.1 \mathrm{MHz}, 55.8 \mathrm{~mW} / \mathrm{cm}^{2}, 50 \%\right.$ duty cycle, pulse wave mode, 3 W, $20 \mathrm{~min}$ ), the nude mice were executed and tumors, brains, lungs, hearts, livers, spleens, kidneys and intestines were harvested and observed using an IVIS spectrum imaging system (PerkinElmer, Waltham, MA, USA) with a DsRed filter (excitation passband: $500-550 \mathrm{~nm}$; emission passband: $575-650 \mathrm{~nm}$ ). Meanwhile, the removed tumor tissues and major organs were fixed with $4 \%$ paraformaldehyde, embedded in paraffin and sectioned at $5 \mu \mathrm{m}$. The cell nuclei were stained with DAPI and then washed 
three times with PBS. The fluorescence intensity was detected to evaluate the distribution of NPs by CLSM. To further investigate the accumulated concentrations of AZD9291 in tissues, the H1975 tumorbearing mice were treated with free AZD9291 via oral gavage and AZD9291-PFCE NPs by intravenous injection at a dose of AZD 9291 ( $5 \mathrm{mg} / \mathrm{kg}$ ), respectively. For the LIFU groups, tumors were triggered by LIFU with the parameters as described above. The mice were sacrificed at $48 \mathrm{~h}$ post treatment. The tumor tissues and major organs (brains, lungs, hearts, livers, spleens, kidneys, muscles and intestines) were collected and weighed. The AZD9291 content in these samples was determined by HPLC.

In vivo therapeutic efficacy. The H1975 tumor-bearing mice were established as mentioned above. When the tumor volume reached $100 \sim 200 \mathrm{~mm}^{3}$, the nude mice were randomly divided into 8 groups $(\mathrm{n}=5)$ : Control, PFCE NPs (0.1 g/kg), AZD9291 (0.5 mg/kg), AZD9291-PFCE NPs (0.5 mg/kg), Control + LIFU, PFCE NPs + LIFU (0.1 g/kg), AZD 9291 + LIFU $(0.5 \mathrm{mg} / \mathrm{kg})$ and AZD9291-PFCE NPs + LIFU $(0.5 \mathrm{mg} / \mathrm{kg})$. Free AZD 9291 was given via gavage every day and other groups received intravenous injection every 3 days. LIFU was applied to the tumor site after different administrations for $4 \mathrm{~h}\left(1.1 \mathrm{MHz}, 55.8 \mathrm{~mW} / \mathrm{cm}^{2}\right.$, $50 \%$ duty cycle, pulse wave mode, $3 \mathrm{~W}, 20 \mathrm{~min}$ ). The mice were treated on days 1, 4, 7,10 and 13. The tumor volume and body weight of the nude mice were monitored every other day. Tumor size was measured by T1-weighted MR imaging and vernier caliper. The tumor growth inhibition (TGI) was determined according to tumor weights. The survival rate of mice had been recorded throughout the experiment. All the nude mice were sacrificed on the 14th day after treatment. The tumors of various groups were resected and subjected to Hematoxylin and Eosin (H\&E) staining and immunohistochemical staining. The proliferation of tumor cells was assessed by anti-Ki67 antibody (ab15580, Abcam). The microvessel density (MVD) of tumor sections was evaluated by CD31 immuno-histochemical staining. Phosphorylated-EGFR ( $p$ EGFR) protein expression status assessed by immunohistochemistry using primary antibodies purchased from Cell Signaling Technology. The sections were observed using an optical microscope. Apoptosis was detected by the TUNEL assay using In Situ Cell Death Detection Kit, according to the manufacturer's instructions and imaged by CLSM.

In vivo biocompatibility studies. $\mathrm{H} 1975$ xenograft-bearing nude mice $(\mathrm{n}=3)$ were used in this study. To estimate the biological safety in vivo, H\&E staining was carried out on the major organs (heart, liver, spleen, lung, kidney, muscle and intestine) at the end of treatment. Furthermore, mice were intravenously injected with $300 \mu \mathrm{L}$ of PFCE NPs or AZD9291-PFCE NPs. At different time points ( 1 and 7 days), blood samples were acquired for electrolytes, blood biochemistry and haematology tests. In addition, to evaluate the biological toxicity of each treatment group, on day 14 , the nude mice were killed and blood and serum samples were harvested for hematological examination, biochemical examination of the liver and renal function.

Statistical analysis. Statistical analyses were performed with GraphPad Prism 8.0 software. All values are presented as mean \pm standard deviation (SD). One-way or two-way analysis of variance (ANOVA) and the Student's $t$ test were applied for statistical evaluation, and differences were considered statistically significant at $p<0.05$. 


\section{Declarations}

Reporting summary. Further information on research design is available in the Nature Research Reporting Summary linked to this article.

\section{Data availability}

The source data underlying all quantitative figures are provided as a Source Data file. All the data supporting the findings of this study are available within the article and its supplementary information files and from the corresponding author upon reasonable request. Source data are provided with this paper.

\section{Acknowledgements}

This work was supported by the National Natural Science Foundation of China $(81627901,81771903)$, Natural Science Foundation of Heilongjiang Province (Grant No. JQ2020H002), the Tou-Yan Innovation Team Program of the Heilongjiang Province (2019-15), China Scholarship Council (No. 201908230103), The National Reform and Development Funds for Local Universities (2020YQ04), National Basic Research Program of China (2015CB931800), and the Key Laboratory of Molecular Imaging Foundation (College of Heilongjiang Province).

\section{Author contributions}

X.S. and L.W. proposed the research direction and guided the project. J.Y., Y.L., X.S. and L.W. conceived the ideas, conducted experiments, analyzed and interpreted data, and wrote the manuscript. J.Y., Y.L., J.S. and Q.X. performed in vivo animal experiments. H.Z. assisted in the data analysis. J.Y., Y.L., R.A., H.W. and X.L. performed the histology experiments and analyzed data. K.W. and L.Y. provided insights in project and experiment design. J.Y. and Y.L. served as co-first authors. All authors reviewed and approved the manuscript.

\section{Competing interests}

The authors declare no competing interests.

\section{Additional information}

Supplementary information is available for this paper at https://doi.org/.

Correspondence and requests for materials should be addressed to L.W. or X.S.

Peer review information Nature Communications thanks reviewer for their contribution to the peer review of this work. Peer reviewer reports are available. 
Reprints and permission information is available at http://www.nature.com/reprints

Publisher's note Springer Nature remains neutral with regard to jurisdictional claims in published maps and institutional affiliations.

\section{References}

1. Arbour, K.C. \& Riely, G.J. Systemic Therapy for Locally Advanced and Metastatic Non-Small Cell Lung Cancer: A Review. JAMA 322, 764-774 (2019).

2. Bray, F.l. et al. Global cancer statistics 2018: GLOBOCAN estimates of incidence and mortality worldwide for 36 cancers in 185 countries. CA Cancer J. Clin. 68, 394-424 (2018).

3. Santos, M.A., Wu, S.K., Regenold, M., Allen, C. \& Hynynen, K. Novel fractionated ultrashort thermal exposures with MRI-guided focused ultrasound for treating tumors with thermosensitive drugs. Science Advances 6, eaba5684 (2020).

4. Tirotta, I. et al. (19)F magnetic resonance imaging (MRI): from design of materials to clinical applications. Chem. Rev. 115, 1106-1129 (2015).

5. Hill, L.K. et al. Protein-Engineered Nanoscale Micelles for Dynamic 19F Magnetic Resonance and Therapeutic Drug Delivery. ACS Nano 13, 2969-2985 (2019).

6. Koshkina, O. et al. Multicore Liquid Perfluorocarbon-Loaded Multimodal Nanoparticles for Stable Ultrasound and 19 F MRI Applied to In Vivo Cell Tracking. Adv. Funct. Mater. 29, 1806485 (2019).

7. Peer, D. et al. Nanocarriers as an emerging platform for cancer therapy. Nature Nanotechnology 2 , 751-760 (2007).

8. Fan, H. et al. A Smart Photosensitizer-Manganese Dioxide Nanosystem for Enhanced Photodynamic Therapy by Reducing Glutathione Levels in Cancer Cells. Angew. Chem. 55, 5477-5482 (2016).

9. Jiang, K. et al. A smart pH-responsive nano-carrier as a drug delivery system for the targeted delivery of ursolic acid: suppresses cancer growth and metastasis by modulating P53/MMP-9/PTEN/CD44 mediated multiple signaling pathways. Nanoscale 9, 9428-9439 (2017).

10. Langereis, S. et al. A Temperature-Sensitive Liposomal 1H CEST and 19F Contrast Agent for MR Image-Guided Drug Delivery. J. Am. Chem. Soc. 131, 1380-1381 (2009).

11. Senders, M.L., Meerwaldt, A.E., Leent, M.M.T.V., Sanchez-Gaytan, B.L. \& Mulder, W.J.M. Probing myeloid cell dynamics in ischaemic heart disease by nanotracer hot-spot imaging. Nature Nanotechnology 15 (2020).

12. Shin, S.H., Park, S.H., Kim, S.W., Kim, M. \& Kim, D. Fluorine MR Imaging Monitoring of Tumor Inflammation after High-Intensity Focused Ultrasound Ablation. Radiology, 171603 (2018).

13. Shin, S.H. et al. Tracking Perfluorocarbon Nanoemulsion Delivery by (19)F MRI for Precise High Intensity Focused Ultrasound Tumor Ablation. Theranostics 7, 562-572 (2017).

14. Kislukhin, A.A. et al. Paramagnetic fluorinated nanoemulsions for sensitive cellular fluorine-19 magnetic resonance imaging. Nat Mater 15, 662-668 (2016). 
15. Temme, S. et al. Noninvasive Imaging of Early Venous Thrombosis by 19F Magnetic Resonance Imaging With Targeted Perfluorocarbon Nanoemulsions. Circulation 131, 1405-1414 (2015).

16. Zhang, L. et al. Size-Modulable Nanoprobe for High-Performance Ultrasound Imaging and Drug Delivery against Cancer. ACS Nano 12, 3449-3460 (2018).

17. Mura, S., Nicolas, J. \& Couvreur, P. Stimuli-responsive nanocarriers for drug delivery. Nature Materials 12, 991-1003 (2013).

18. Karimi, M. et al. Smart micro/nanoparticles in stimulus-responsive drug/gene delivery systems. Chem. Soc. Rev. 45, 1457-1501 (2016).

19. Cao, Y. et al. Drug Release from Phase-Changeable Nanodroplets Triggered by Low-Intensity Focused Ultrasound. Theranostics 8, 1327-1339 (2018).

20. Zhang, Y., Yu, J., Bomba, H.N., Zhu, Y. \& Gu, Z. Mechanical Force-Triggered Drug Delivery. Chem. Rev. $116,12536-12563$ (2016).

21. Lyon, P.C. et al. Safety and feasibility of ultrasound-triggered targeted drug delivery of doxorubicin from thermosensitive liposomes in liver tumours (TARDOX): a single-centre, open-label, phase 1 trial. Lancet Oncol. 19, 1027-1039 (2018).

22. Szablowski, J.O., Barzion, A. \& Shapiro, M.G. Achieving Spatial and Molecular Specificity with Ultrasound-Targeted Biomolecular Nanotherapeutics. Acc. Chem. Res. 52, 2427-2434 (2019).

23. Airan, R.D. et al. Noninvasive Targeted Transcranial Neuromodulation via Focused Ultrasound Gated Drug Release from Nanoemulsions. Nano Lett. 17, 652-659 (2017).

24. Ozdas, M.S. et al. Non-invasive molecularly-specific millimeter-resolution manipulation of brain circuits by ultrasound-mediated aggregation and uncaging of drug carriers. Nature communications 11, 4929 (2020).

25. Abrahao, A. et al. First-in-human trial of blood-brain barrier opening in amyotrophic lateral sclerosis using MR-guided focused ultrasound. Nature communications 10, 4373 (2019).

26. Wang, M. et al. Multifunctional Nanoparticles for Multimodal Imaging-Guided Low-Intensity Focused Ultrasound/Immunosynergistic Retinoblastoma Therapy. ACS Applied Materials \& Interfaces 12, 5642-5657 (2020).

27. Zhao, H. et al. Cell-penetrating Peptide-modified Targeted Drug-loaded Phase-transformation Lipid Nanoparticles Combined with Low-intensity Focused Ultrasound for Precision Theranostics against Hepatocellular Carcinoma. Theranostics 8, 1892-1910 (2018).

28. Rapoport, N., Gao, Z. \& Kennedy, A. Multifunctional Nanoparticles for Combining Ultrasonic Tumor Imaging and Targeted Chemotherapy. J. Natl. Cancer Inst. 99, 1095-1106 (2007).

29. Soria, J. et al. Osimertinib in untreated EGFR-mutated advanced non-small-cell lung cancer. The New England Journal of Medicine 378, 113-125 (2018).

30. Winter, P.M. et al. Molecular imaging of angiogenesis in nascent $\mathrm{Vx}-2$ rabbit tumors using a novel alpha(nu)beta3-targeted nanoparticle and 1.5 tesla magnetic resonance imaging. Cancer Res. 63, 5838-5843 (2003). 
31. Winter, P.M. et al. Minute dosages of alpha(nu)beta3-targeted fumagillin nanoparticles impair Vx-2 tumor angiogenesis and development in rabbits. FASEB J. 22, 2758-2767 (2008).

32. Pan, D. et al. A strategy for combating melanoma with oncogenic c-Myc inhibitors and targeted nanotherapy. Nanomed. Nanotechnol. Biol. Med. 10, 241-251 (2015).

33. Xu, X. et al. Folate receptor-targeted (19) F MR molecular imaging and proliferation evaluation of lung cancer. J. Magn. Reson. Imaging 48, 1617-1625 (2018).

34. Kaneda, M.M., Caruthers, S.D., Lanza, G.M. \& Wickline, S.A. Perfluorocarbon Nanoemulsions for Quantitative Molecular Imaging and Targeted Therapeutics. Ann. Biomed. Eng. 37, 1922-1933 (2009).

35. Wu, L. et al. Local Intratracheal Delivery of Perfluorocarbon Nanoparticles to Lung Cancer Demonstrated with Magnetic Resonance Multimodal Imaging. Theranostics 8, 563-574 (2018).

36. Malachowski, K. et al. Stimuli-Responsive Theragrippers for Chemomechanical Controlled Release. Angew. Chem. 53, 8045-8049 (2014).

37. Zhu, D. et al. Remotely controlled opening of delivery vehicles and release of cargo by external triggers. Adv. Drug Del. Rev. 138, 117-132 (2019).

38. Yeom, J. et al. Chiral Supraparticles for Controllable Nanomedicine. Adv. Mater. 32, 1903878 (2020).

39. Li, Y. et al. Tumor-Specific Multiple Stimuli-Activated Dendrimeric Nanoassemblies with Metabolic Blockade Surmount Chemotherapy Resistance. ACS nano 11, 416-429 (2017).

40. Benien, P. \& Swami, A. 3D tumor models: history, advances and future perspectives. Future Oncol. 10, 1311-1327 (2014).

41. Wang, X. et al. Delivery of platinum(IV) drug to subcutaneous tumor and lung metastasis using bradykinin-potentiating peptide-decorated chitosan nanoparticles. Biomaterials 35, 6439-6453 (2014).

42. Cross, D. et al. AZD9291, an irreversible EGFR TKI, overcomes T790M-mediated resistance to EGFR inhibitors in lung cancer. Cancer Discov. 4, 1046-1061 (2014).

43. Shi, Y. et al. Complete Regression of Xenograft Tumors upon Targeted Delivery of Paclitaxel via $\Pi-\Pi$ Stacking Stabilized Polymeric Micelles. ACS Nano 9, 3740-3752 (2015).

44. Zhao, H. et al. Cell-penetrating Peptide-modified Targeted Drug-loaded Phase-transformation Lipid Nanoparticles Combined with Low-intensity Focused Ultrasound for Precision Theranostics against Hepatocellular Carcinoma. Theranostics 8, 1892-1910 (2018).

45. Bulte, J.W.M. et al. MR cholangiography demonstrates unsuspected rapid biliary clearance of nanoparticles in rodents: Implications for clinical translation. Nanomed. Nanotechnol. Biol. Med. 10, 1385-1388 (2014).

\section{Figures}




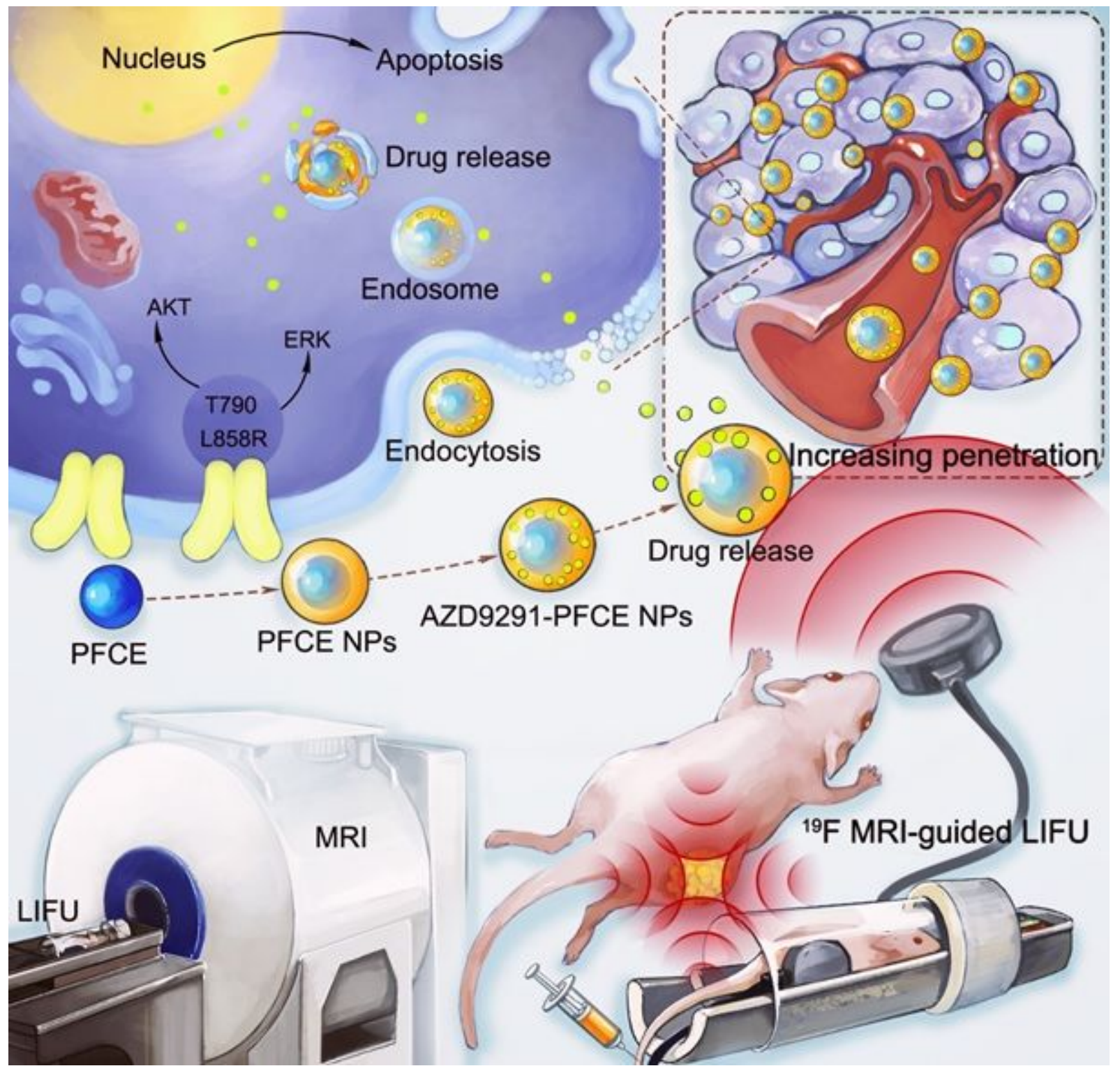

Figure 1

Schematic illustration of 19F MRI-guided and LIFU-triggered drug delivery systems promoting efficient delivery and deep penetration for EGFR-targeted therapy in NSCLC. Optimized AZD9291-PFCE NPs reach high accumulation in NSCLC tumor tissue. LIFU specifically triggered on-demand drug release of AZD 9291-PFCE NPs and further increased tumor penetration and cellular internalization. Furthermore, the biodistribution of NPs could be tracked with 19F MRI in a quantitative manner, providing guidance of precise treatment. 
a

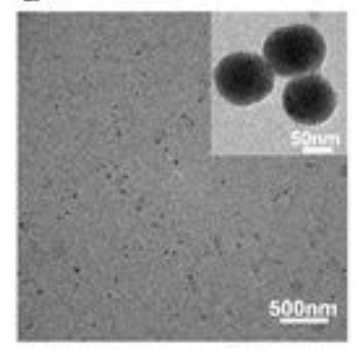

b

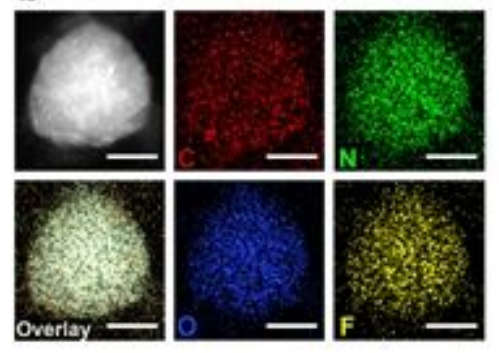

e
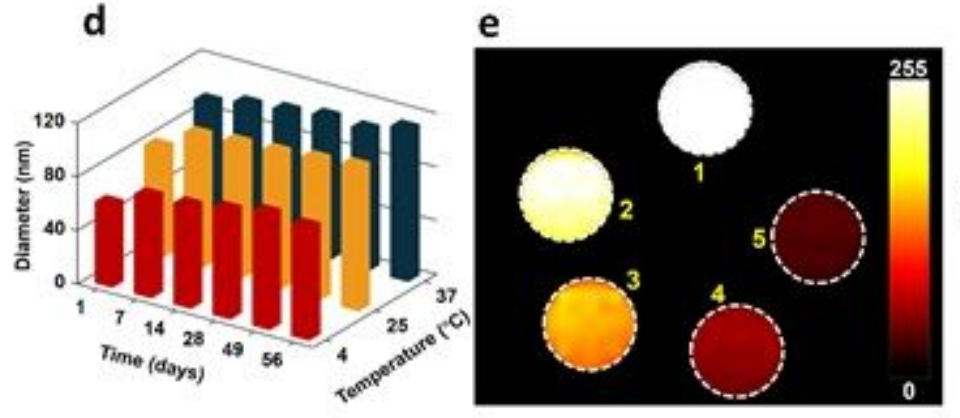

h
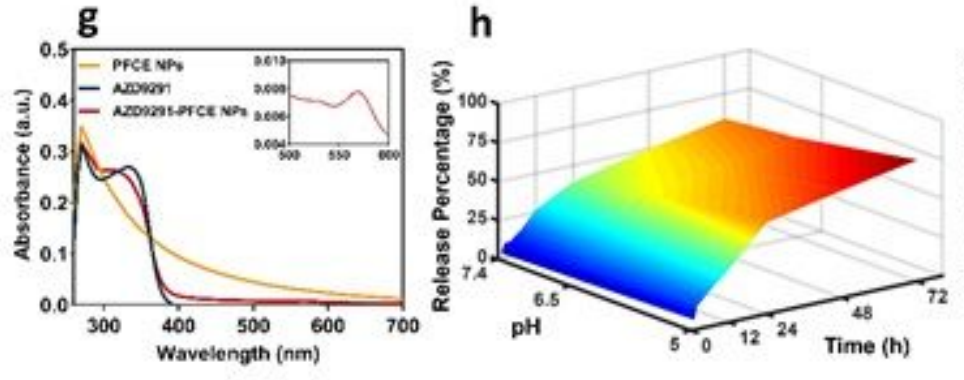
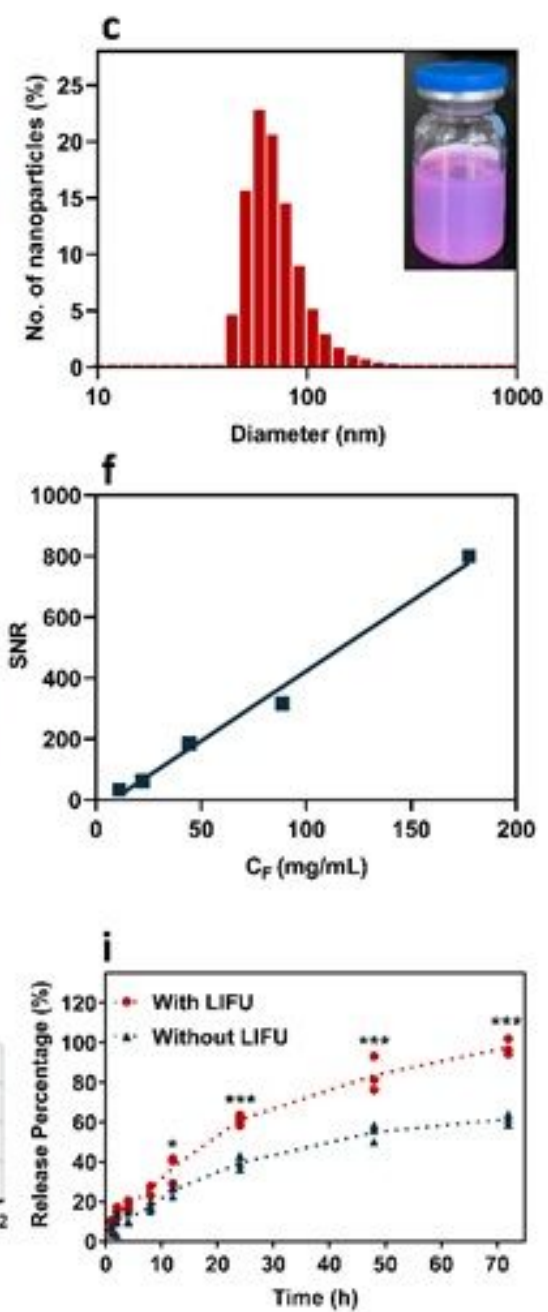

Figure 2

Characterization of AZD9291-PFCE NPs. a TEM image of AZD9291-PFCE NPs. b Dark-field image and the corresponding EDS elemental mapping of carbon (C), nitrogen $(N)$, oxygen $(0)$ and fluorine $(F)$. Scale bar is $100 \mathrm{~nm}$. c Average hydrodynamic size of AZD9291-PFCE NPs characterized by dynamic light scattering (DLS) and digital photograph (inset). d Size stability test of AZD9291-PFCE NPs at $4^{\circ} \mathrm{C}, 25^{\circ} \mathrm{C}$ and $37^{\circ} \mathrm{C}$ for up to 8 weeks. e 19F MRI of AZD9291-PFCE NPs with various $19 \mathrm{~F}$ concentrations (CF). (15): $177.5,88.75,44.38,22.19,11.09 \mathrm{mg} / \mathrm{mL}$. f Plot of the 19F MRI signal-to-noise ratio (SNR) versus $19 \mathrm{~F}$ concentration. g UV-Vis absorption spectra of PFCE NPs, AZD9291 and AZD9291-PFCE NPs. Inset photos indicate the typical UV absorption peaks of AZD9291-PFCE NPs labeled with rhodamine. $h$ AZD9291 release characteristics of AZD9291-PFCE NPs in PBS at pH 5.0, 6.5, or 7.4 within 72 hours. i AZD9291 release profiles of AZD9291-PFCE NPs in PBS triggered by LIFU at pH $7.4(n=3 ; * P<0.05, * \star \star P<0.001)$. 
a

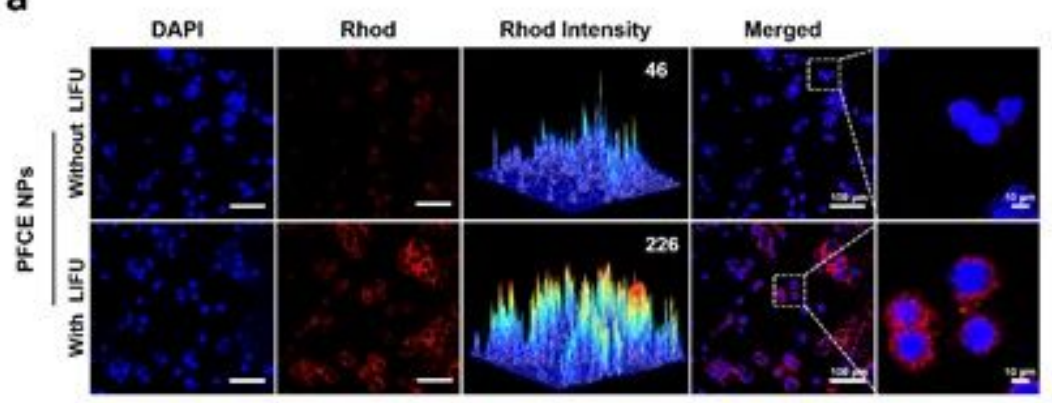

DAPI

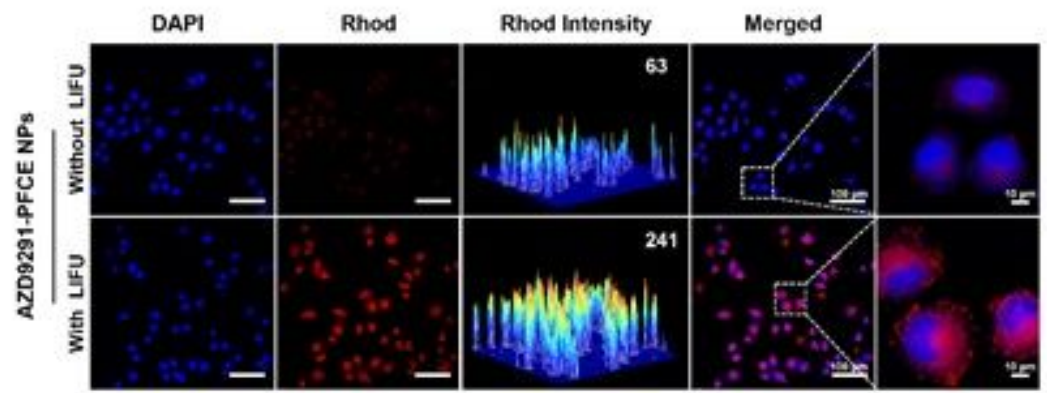

d
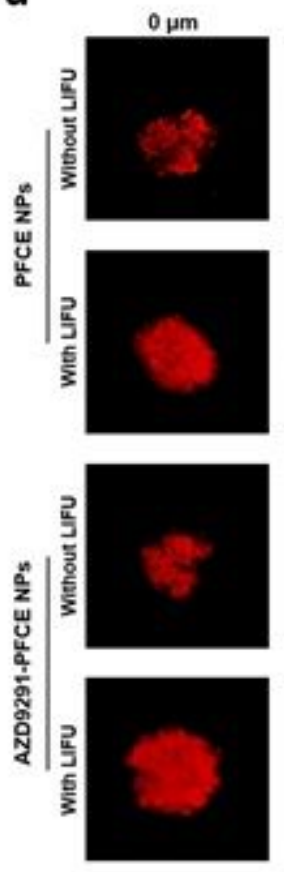

$20 \mu \mathrm{m}$
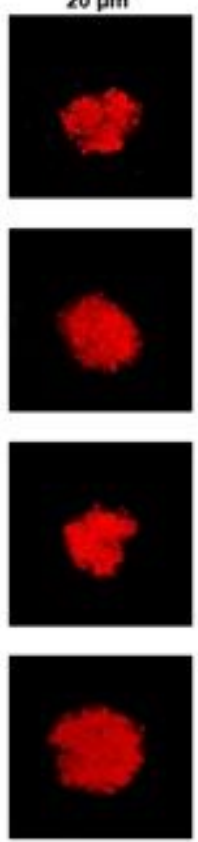

$40 \mu \mathrm{m}$

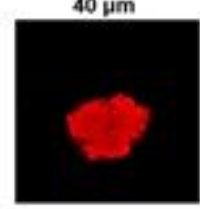

$60 \mu \mathrm{m}$
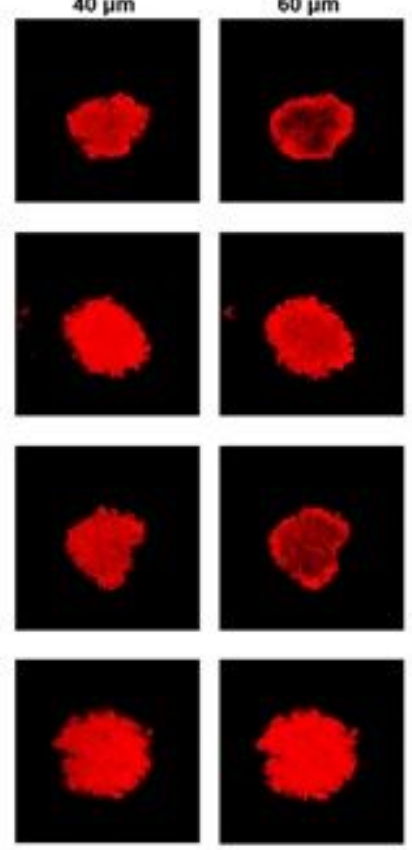

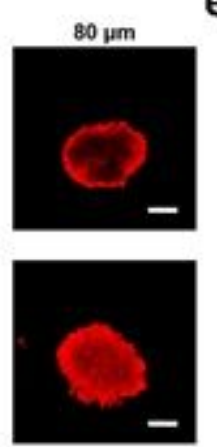

e

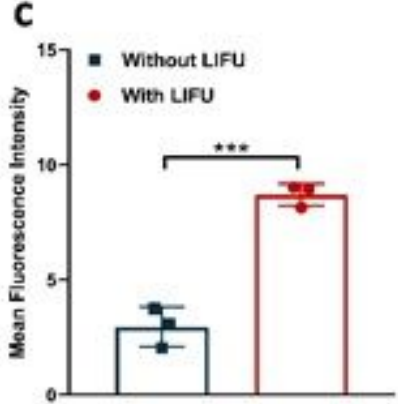

AZDe291-PFCE NPS

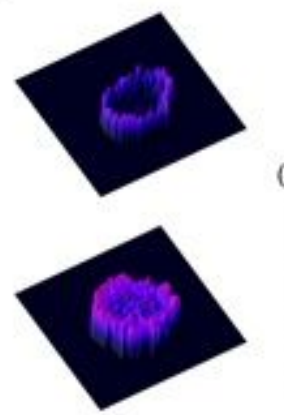

(a.u.)
200
180
160
140
120
100
80
60
40
20
0

Figure 3

Cellular uptake and tumor penetration of NPs in vitro. a CLSM images of H1975 cells incubated with PFCE NPs or AZD9291-PFCE NPs with and without LIFU irradiation for $4 \mathrm{~h}$. Blue fluorescence indicates nuclei stained with DAPl; red fluorescence represents rhodamine-labeled PFCE NPs or AZD9291-PFCE NPs and Rhod intensity represents the total integrated intensity of red fluorescence acquired by calculating the integral optical density (IOD); Scale bar is $100 \mu \mathrm{m}$. b-c Mean fluorescence intensity (MFI) quantification of H1975 cells treated with PFCE NPs (b) or AZD9291-PFCE NPs (c) with and without LIFU 
irradiation for $4 \mathrm{~h}$. d CLSM images showing in vitro penetration of rhodamine-labeled PFCE NPs or AZD 9291-PFCE NPs with and without LIFU irradiation in 3D-cultured H1975 tumor spheroids. Scale bar = $200 \mu \mathrm{m}$. The Z-stack scanning for the spheroids was performed at $10 \mu \mathrm{m}$ intervals by CLSM. e 3D diagram of $80 \mu \mathrm{m}$ depth sections of tumor spheroids in (d) reconstructed by Image J. Data are presented as mean \pm standard deviation $(n=3)$, ** $P<0.01$, *** $P<0.001$.
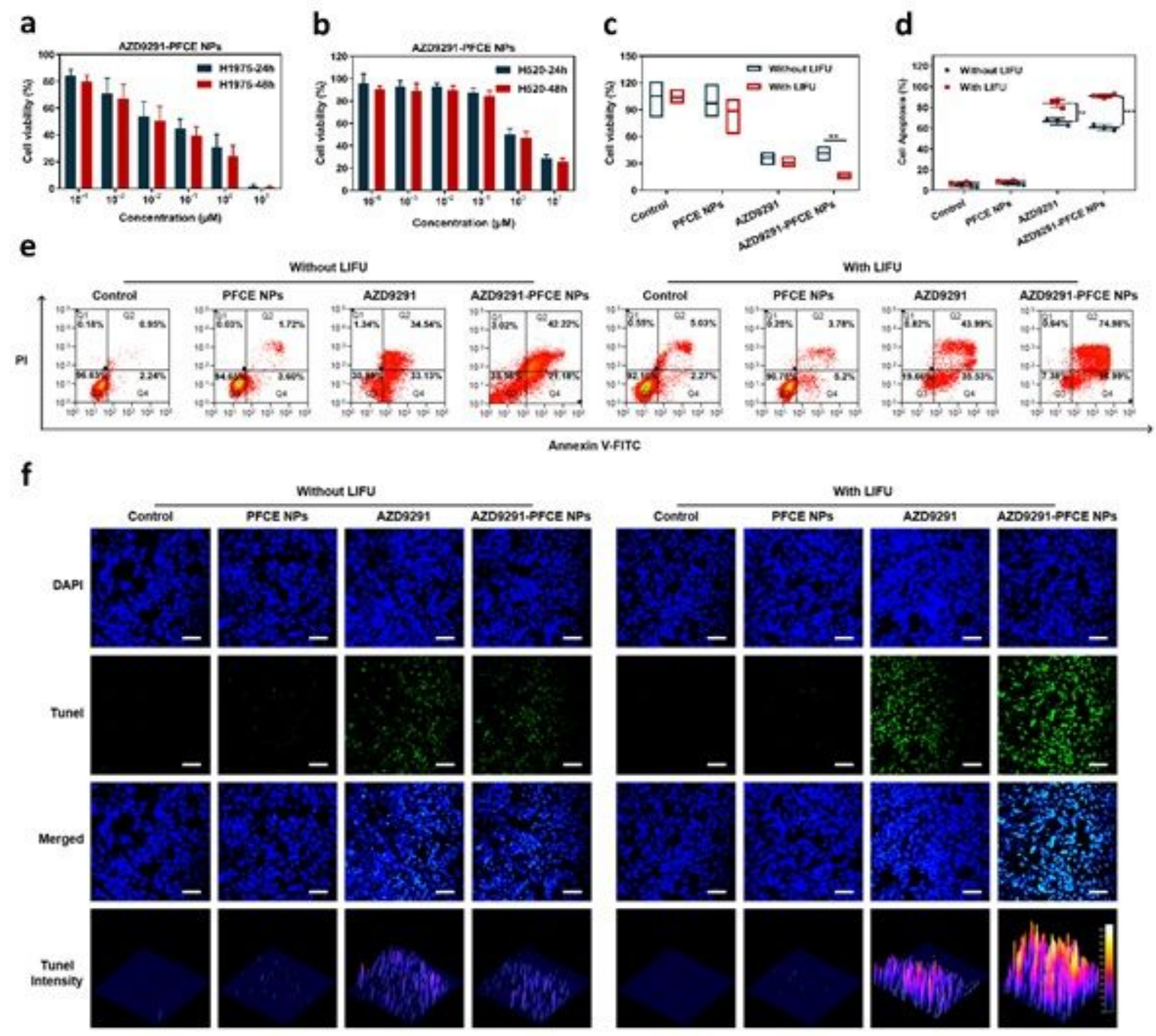

Figure 4

Antitumor effect in vitro. a-b Cell viability of H1975 and H520 cells treated with AZD9291-PFCE NPs at various AZD 9291 concentrations for $24 \mathrm{~h}$ and $48 \mathrm{~h}$. c Cell viability of $\mathrm{H} 1975$ cells after incubation with different formulations for $24 \mathrm{~h}$ with or without LIFU irradiation (1.1 MHz; $55.8 \mathrm{~mW} / \mathrm{cm} 2 ; 15 \mathrm{~min})$. d Quantitative analysis of cell apoptosis. e Cell apoptosis analysis by flow cytometry in $\mathrm{H} 1975$ cells on different treatments for $24 \mathrm{~h}$. $\mathrm{f}$ Cell apoptosis in $\mathrm{H} 1975$ cells assessed by TUNEL staining after various treatments for $24 \mathrm{~h}$. Scale bar is $100 \mu \mathrm{m}$. Data are shown as mean $\pm S D(n=3)$. ${ }^{\star \star} P<0.05, * \star \star P<0.001$. 
a
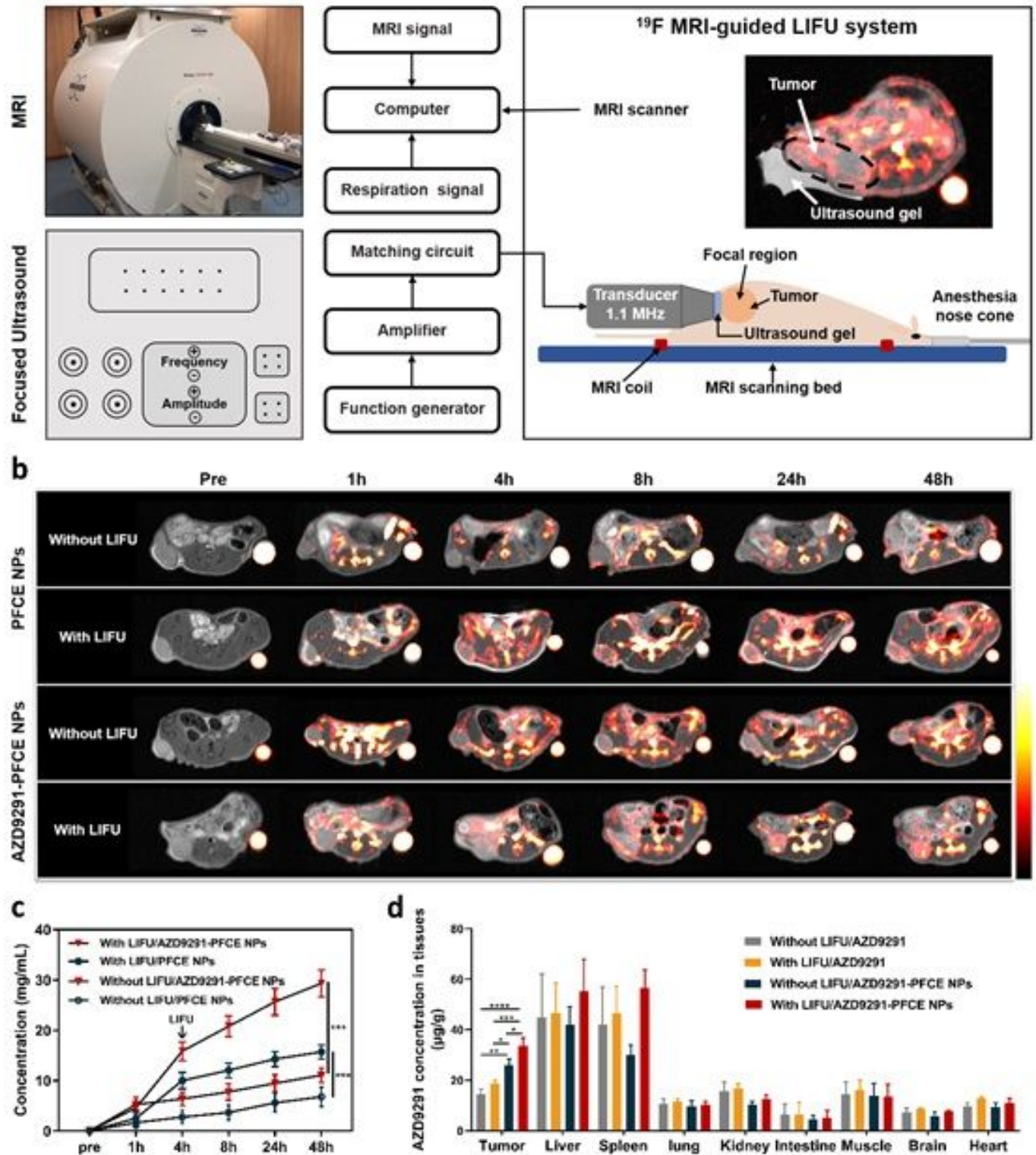

e

f
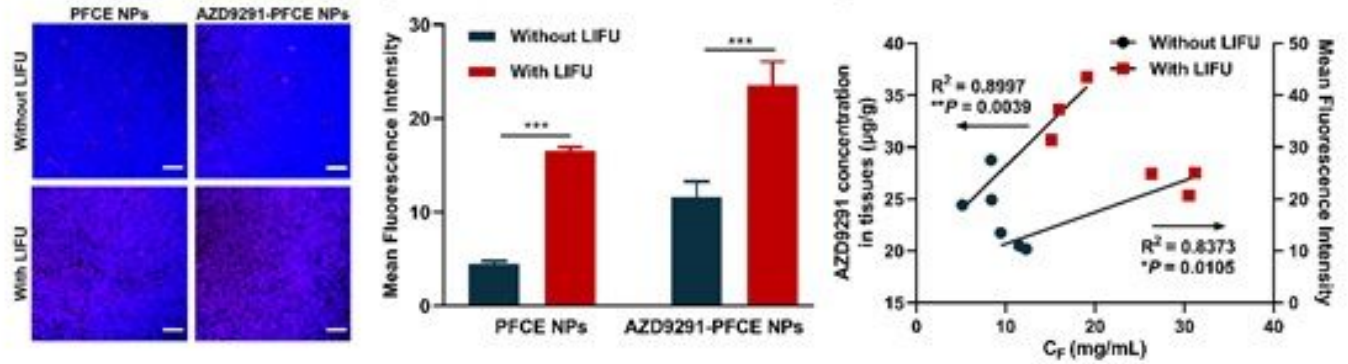

Figure 5

19F MRI-guided flexible LIFU for increasing penetration and accumulation of NPs in tumor tissues. a Schematic illustration of the experimental setup. Axial 19F MR image of H1975 tumor-bearing mice after intravenous injection of AZD9291-PFCE NPs within the MRI-compatible LIFU setup (inset). b 1H/19F merged images of mice injected with $300 \mu \mathrm{L}$ of PFCE NPs and AZD9291-PFCE NPs at different time points. Four hours after injection of NPs, LIFU (1.1 MHz, 55.8 mW/cm2, $20 \mathrm{~min})$ was applied to an area 
covering the tumor and MRI was performed synchronously. c Quantification of NPs accumulated in tumors. The increased accumulation was visualized after LIFU sonication. d AZD9291 concentration in tumor tissues and major organs at 48h after treatment with free AZD9291 and AZD9291-PFCE NPs measured by HPLC. e Rhodamine-labeled PFCE NPs and AZD9291-PFCE NPs distribution in tumor tissues. Nuclei were stained by DAPI. Scale bar is $100 \mu \mathrm{m}$. $\mathrm{f}$ Quantitative analysis of the mean fluorescence intensity of PFCE NPs and AZD9291-PFCE NPs in tumor tissues. g Linear regression analysis of AZD9291 concentration, mean fluorescence intensity and 19F concentrations (CF) in tumors. Data are shown as mean $\pm S D(n=3 ; * P<0.05$, ** $P<0.01$, *** $P<0.001$, **** $P<0.0001)$.

a

b
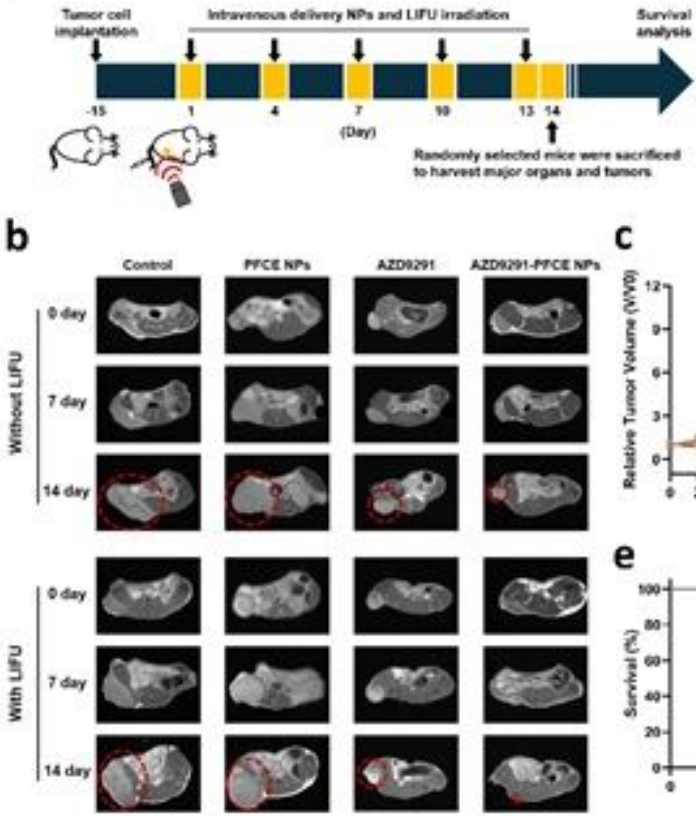

g
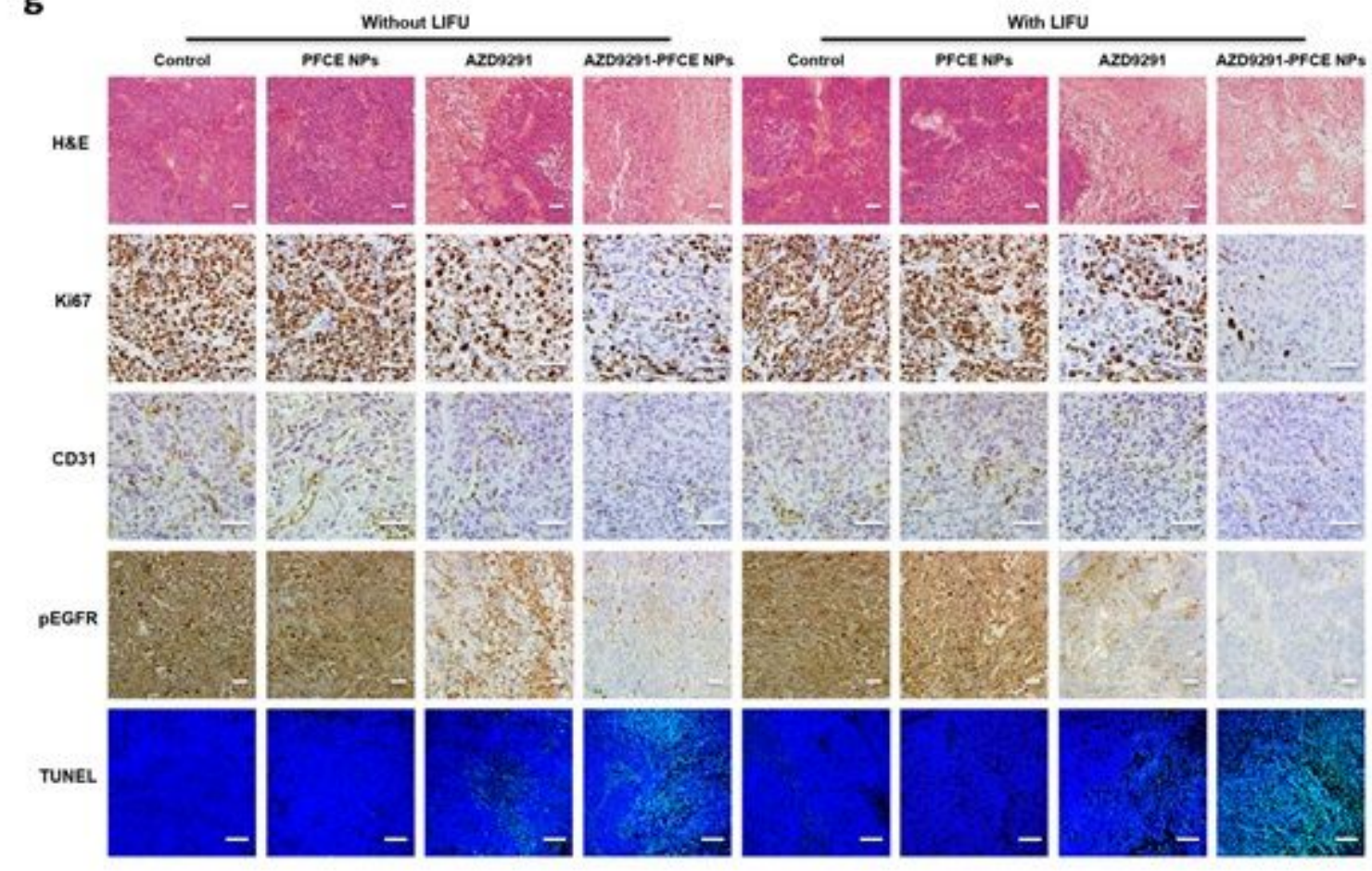

C
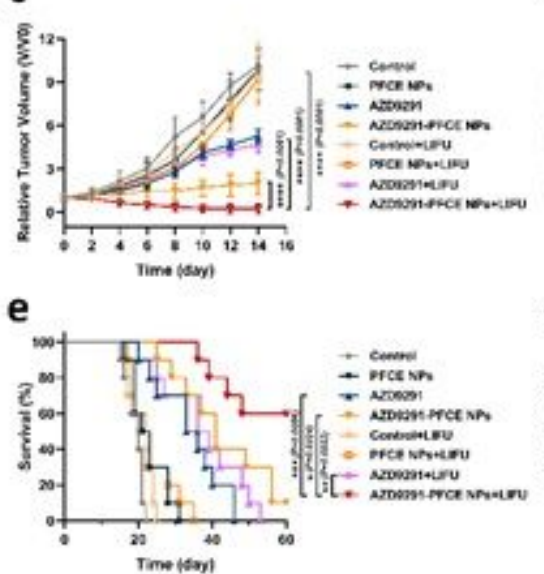

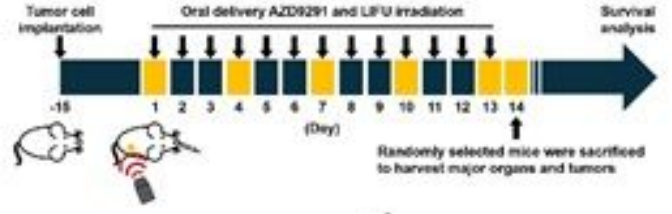

d f
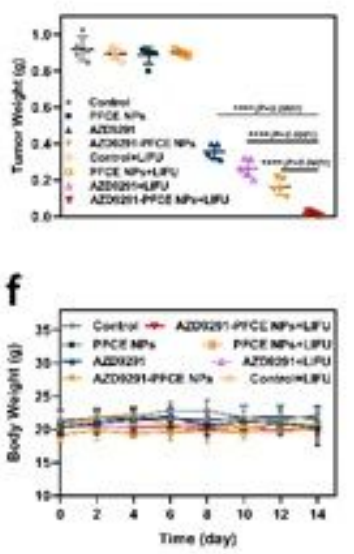


\section{Figure 6}

Antitumor efficiency in H1975 tumor-bearing mice model. a Treatment schemes. H1975 cells were implanted into the right thigh of mice ( $\mathrm{n}=5$ per group). The mice were administrated with different treatment groups, Control, PFCE NPs (0.1 g/ kg), AZD 9291 (0.5 mg/kg), AZD9291-PFCE NPs (0.5 mg/kg), Control+LIFU, PFCE NPs+LIFU (0.1 g/kg), AZD9291+LIFU $(0.5 \mathrm{mg} / \mathrm{kg})$ and AZD9291-PFCE NPs+LIFU (0.5 $\mathrm{mg} / \mathrm{kg}$ ). Free AZD9291 was given via gavage every day and other groups received intravenous injection every 3 days. LIFU was applied to the tumor site after different administrations for $4 \mathrm{~h}(1.1 \mathrm{MHz}, 55.8$ $\mathrm{mW} / \mathrm{cm} 2,20 \mathrm{~min}$ ). b Tumor progression (red dashed circles) of $\mathrm{H} 1975$ tumor-bearing mice model monitored with T1-weighted MRI of mice from each group on day 0,7 and $14 \mathrm{~d}$ after various treatment. c The relative tumor growth curves of $\mathrm{H} 1975$ tumor-bearing mice with different treatments $(n=5)$. $d$ The weight of tumors excised from mice on day $14(n=5)$. e Survival curves of the mice with different treatment $(n=10)$. f Body weight changes of H1975 tumor-bearing mice with different treatments $(n=5)$. $\mathrm{g}$ H\&E staining, immunohistochemical analysis, and TUNEL assay of tumor sections on day 14 after treatment. The scale bar is $100 \mu \mathrm{m}$. The results are presented as mean \pm standard deviation. ${ }^{*} P<0.05$, ** $\mathrm{P}<0.01, * \star * \mathrm{P}<0.001, * \star \star * \mathrm{P}<0.0001$.
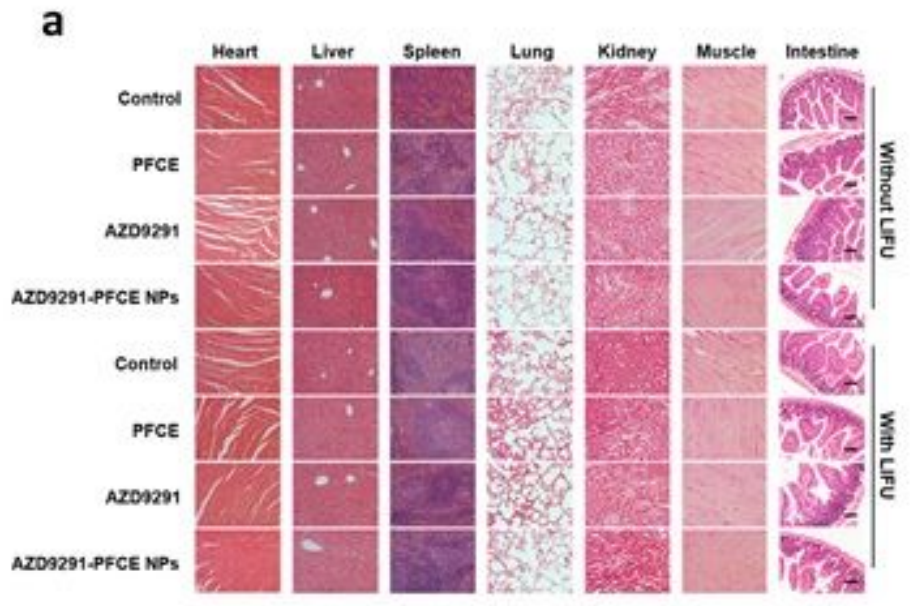

d
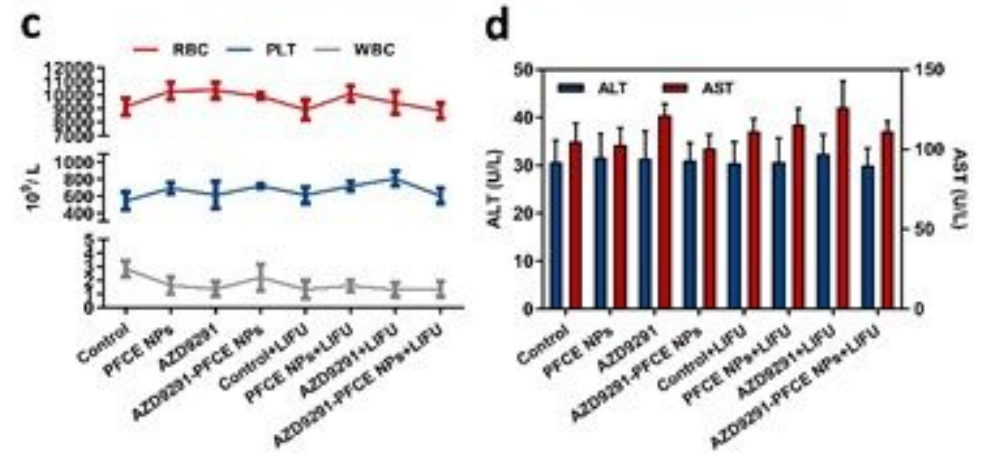

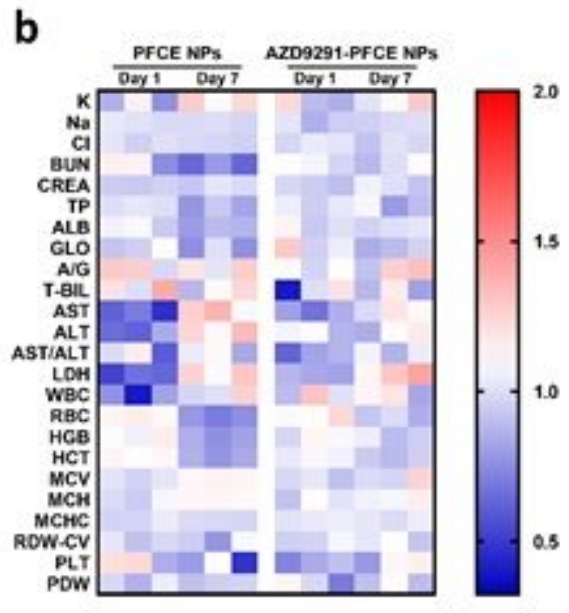

e

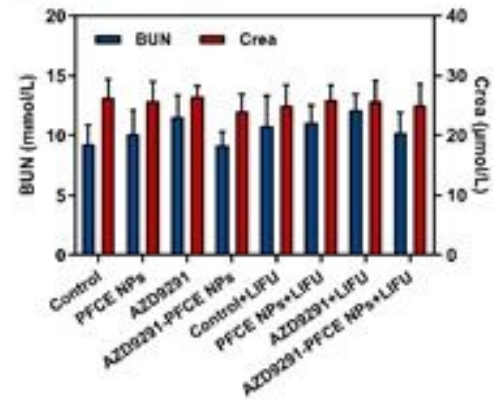

Figure 7

Biosafety assessment in vivo. a H\&E staining in major organs (heart, liver, spleen, lung, kidney, muscle and intestine) of all groups after treatments. The scale bars are $100 \mu \mathrm{m}$. b Electrolytes, blood biochemistry and haematologic indexes of mice intravenously injected with PFCE NPs or AZD9291-PFCE 
NPs. The experiment was performed on days 1 and 7 after injection $(n=3)$. c Hematological examination of RBC, PLT and WBC, d liver function indicators ALT and AST and e renal function indicators BUN and Crea from the mice treated with different formulations $(n=3)$.

\section{Supplementary Files}

This is a list of supplementary files associated with this preprint. Click to download.

- SupportingInformation.docx 